\title{
Health warning labels and alcohol selection: a randomised controlled experiment in a naturalistic shopping laboratory
}

\author{
Natasha Clarke' (D, Anna K. M. Blackwell ${ }^{2}$ (D) Katie De-loyde ${ }^{2}$ (D), Emily Pechey' (D), \\ Alice Hobson' (D, Mark Pilling' (D, Richard W. Morris ${ }^{3}$, Theresa M. Marteau' (D) \& \\ Gareth J. Hollands'
}

Behaviour and Health Research Unit, Department of Public Health and Primary Care, University of Cambridge, Cambridge, UK, Tobacco and Alcohol Research Group, School of Psychological Science, University of Bristol, Bristol, UK² and Bristol Medical School, University of Bristol, Bristol, UK ${ }^{3}$

\begin{abstract}
Background and aims Health warning labels (HWLs) on tobacco products reduce smoking. There is an absence of evidence concerning the impact of alcohol HWLs on selection or purchasing in naturalistic settings. Using a commercial-standard naturalistic shopping laboratory, this study aimed to estimate the impact on selection of alcoholic drinks of HWLs describing adverse health consequences of excessive alcohol consumption. Design A between-subjects randomised experiment with three groups was conducted: group 1: image-and-text HWL; group 2: text-only HWL; group 3: no HWL. Setting A commercial-standard naturalistic shopping laboratory in the United Kingdom. Participants Adults $(n=399,55 \%$ female) over the age of 18 years, who purchased beer or wine weekly to drink at home. Interventions Participants were randomised to one of three groups varying in the HWL displayed on the packaging of the alcoholic drinks: (i) image-and-text HWL ( $n=135)$; (ii) text-only HWL $(n=129)$; (iii) no HWL $(n=135)$. Participants completed a shopping task, selecting items from a range of alcoholic and non-alcoholic drinks, and snacks. Measurement The primary outcome was the proportion of alcoholic drinks selected. Secondary outcomes included HWL ratings on negative emotional arousal and label acceptability. Findings There was no clear evidence of a difference in the HWL groups for the percentage of drinks selected that were alcoholic compared to no HWL (44\%): image-and-text HWL: 46\% (odds ratio $[\mathrm{OR}]=1.08,95 \%$ confidence interval $[\mathrm{CI}]=0.82,1.42$ ); text-only HWL: 41\% $(\mathrm{OR}=0.87,95 \% \mathrm{CI}=0.67,1.14)$. Concordant with there being no difference between groups, there was extreme evidence in favour of the null hypothesis (Bayes factor $[\mathrm{BF}]<0.01$ ). Negative emotional arousal was higher $(P<0.001)$ and acceptability lower $(P<0.001)$ in the image-and-text HWL group, compared to the text-only HWL group. Conclusions In a naturalistic shopping laboratory, there was no evidence that health warning labels describing the adverse health consequences of excessive alcohol consumption changed selection behaviour.
\end{abstract}

Keywords Alcohol, choice architecture, graphic warnings, health warning label, labelling, pictorial health warning label.

Correspondence to: Natasha Clarke, Behaviour and Health Research Unit, Institute of Public Health, University of Cambridge, Cambridge, UK.

E-mail: ncc42@medschl.cam.ac.uk

Submitted 3 September 2020; initial review completed 15 December 2020; final version accepted 7 April 2021

\section{INTRODUCTION}

Excessive consumption of alcohol causes a significant population health burden, increasing the risk of a range of diseases including liver disease, heart disease and many cancers [1,2]. An objective of the "Global Strategy to Reduce the Harmful Use of Alcohol" is to reduce harmful use by $10 \%$ by 2025 [3]. One potential intervention to discourage excessive alcohol consumption is the use of health warning labels (HWLs) on packaging. There is strong evidence that HWLs on tobacco are both effective and a feasible population-level intervention [4,5], with larger effects of image-and-text HWLs than text-only HWLs [6-8], and similar effects evident among those in more and less deprived groups [9]. Given this, their potential application to alcohol 
products has been suggested by academics and public health bodies [10-13], although such bodies have also highlighted that caution should be applied when comparing evidence across products [11].

In the United Kingdom, the only mandatory health information required on alcohol product labels is alcohol strength by volume (ABV), the net quantity of the drink, and allergens [14]. There are further best-practice recommendations to include three key elements: unit content, pregnancy warnings and a link to the industry-funded Drinkaware website [15]. However, even if this voluntary information is included labels are typically small, placed on the back of product packaging and may attract only minimal consumer attention in their current form [16]. These factors probably contribute to the ineffectiveness of current alcohol labelling [17]. Elements that may increase label effectiveness include larger labels and health warnings beyond those aimed at pregnant women [18]. Providing more highly visible health risks on labels may increase the currently low awareness of harms, such as the link between alcohol and cancer [19].

Evidence from online studies suggests that HWLs can reduce hypothetical selection of alcoholic drinks, with the addition of an image increasing effectiveness, an effect which may be mediated by negative emotional arousal [20]. There is limited evidence available in laboratory settings for the effectiveness of HWLs, with only one study to date looking at consumption [21]. Initial studies suggest that both image-and-text and text-only HWLs are effective at decreasing consumption rate [21], with image-and-text HWLs exerting larger effects on consumption intentions [22]. However, HWLs with images are also the least acceptable [20], and evidence suggests they may be less acceptable on alcohol products than they are on food products [23]. A lack of awareness of the health risks associated with alcohol may contribute to the low acceptability of HWLs, but acceptability can be increased by raising awareness of harms associated with drinking [24].

Importantly, there is an absence of evidence on the impact of both text-only and image-and-text HWLs on alcohol selection or consumption in naturalistic settings more likely to reflect real-world alcohol-related behaviour [25]. Assessment of this is particularly key, given that effect sizes observed within preliminary online studies may not translate to laboratory and field settings [25]. Naturalistic settings - such as bar laboratories - are frequently used in alcohol consumption research to mimic typical drinking environments [26]. Recently, artificial shopping laboratories have been used to study tobacco $[27,28]$ and sugar-sweetened beverage purchasing behaviour, with HWLs reducing the likelihood of purchasing these drinks [29]. Such settings provide greater ecological validity than studies conducted online. Moreover, they offer an alternative for testing interventions — such as aversive product labels - where a lack of palatability to commercial stakeholders is a barrier to identifying viable field settings.

The current study is the first to use a naturalistic shopping laboratory (Shopper Lab) to investigate the impact of alcohol HWLs on selection behaviour. The primary aim of the study was to estimate the impact on selection of alcoholic drinks displaying image-and-text or text-only HWLs describing adverse health consequences of excessive alcohol consumption. It was hypothesised that image-and-text and text-only HWLs placed on bottles and cans of beer and wine would reduce their selection compared to no HWLs. Secondary aims were (1) to assess the impact of image-and-text and text-only HWLs on alcohol quasi-purchasing behaviour (i.e. total and proportional spend on alcohol and alcohol units bought) and (2) to describe emotional responses to, and acceptability of, imageand-text and text-only HWLs.

\section{METHODS}

The study protocol and a detailed statistical analysis plan were pre-registered (ISRCTN18275963; OSF:https://osf. io/hbdg3/).

Design

The study used a between-subjects design. Participants were randomly allocated to one of three possible experimental groups: Group 1: image-and-text HWL; Group 2: text-only HWL; Group 3: no HWL (Box 1).

\section{Randomisation}

Stock in the shopping laboratory could only be changed at most twice a day, and could not be re-configured for every participant. Participants were therefore randomised to attend an experimental session consistent with their group allocation. Sessions were allocated using stratified randomisation and participants were allocated using simple randomisation. The statistician conducting the randomisation was blind to group assignment. Allocation was double-blind, with neither the recruiter nor the participants aware of the intervention assignment; although the recruiter was aware that the participant was in group A, B or $\mathrm{C}$, they were unaware of to which label group this corresponded. See Supporting information, S1, Figs S1 and S2) for further randomisation details.

\section{Setting}

The study was conducted in a naturalistic shopping laboratory setting in February 2020 (Blue Yonder Shopper Lab). This is a $4 \times 7.6 \mathrm{~m}$ room, designed to mimic a real supermarket store, and is used specifically for commercial research purposes. Products were branded and prices 
Box 1. Study design.

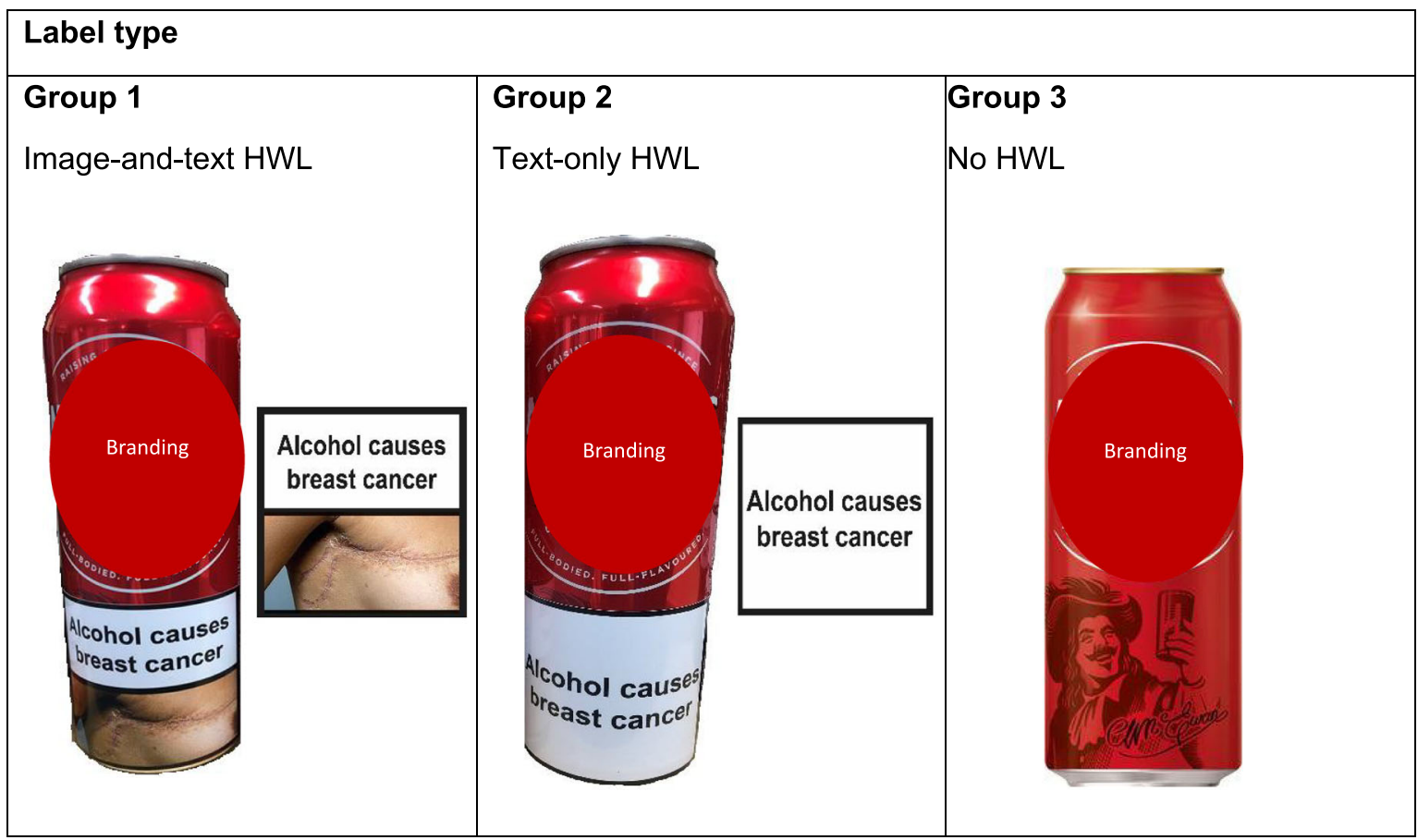

N.B. HWLs were displayed on branded products on top of usual product packaging (see supplementary material Figure 55 for more example images).

consistent with those in UK stores. The Shopper Lab displayed drinks (alcoholic and non-alcoholic) and snacks with product selection based on current supermarket stock in English stores, except for non-alcoholic beer and wine, for which more options were available than are usually provided. Shelves were re-stocked after each participant session. Further details and photographs of the set-up can be found in the Supporting information, S3, Figs S3 and S4.

\section{Participants}

Participants were adults (18+) residing in the United Kingdom, who consumed beer or wine regularly (i.e. at least once a week), purchased supermarket products (food and drink) for their household and shopped in a physical store at least monthly. Participants attended the study in person and were recruited via Blue Yonder Research Ltd; the agency has a pool of approximately 10000 members with a wide range of social characteristics.

Based on previous research on alcohol HWLs [20], a sample was recruited to detect a difference of at least 16 percentage points for the proportion of participants selecting an alcoholic beverage between no HWL and the HWL groups [80\% power, $\alpha=0.025$ (applying Bonferroni adjustment for two comparisons to the no HWL group)]. At least 130 participants per group was required (390 participants in total). Given uncertainty concerning the translation of effect sizes seen in online settings to realworld laboratory settings, a larger sample size would have been preferable. However, there was no prior comparable study to aid us in estimating effect sizes, and available resources also dictated that this represented the largest possible sample size.

\section{Interventions}

\section{Health warning labels}

Depending on the allocated group, the alcoholic products either displayed HWLs (image-and-text or text-only) or no HWLs, applied directly on the product. The same products were used in each group. Within the HWL groups, different variants of HWLs (i.e. type of health consequence described on the HWL) were used to increase variety, maximise engagement and increase potential impact of at least one HWL across our sample. This was also informed by tobacco guidelines specifying that rotating warnings are most effective. Image-and-text and text-only HWL variants described the same six health consequences. For example, both included a breast cancer HWL and a liver cancer HWL. The specific warnings used were created by a graphic designer and were based on previous studies highlighting these HWLs as being most effective in 
increasing negative emotions [23] and decreasing the odds of selecting alcohol [20]. Illustrative examples of labelled alcohol products are included in the Supporting information, S5, Fig. S5 and the full range of labels are available at https://osf.io/gye $5 n /$.

Labelling interventions are classed as 'Information' interventions that focus on the 'Product' of interest in the TIPPME intervention typology (Typology of Interventions in Proximal Physical Micro-Environments [30]).

\section{Outcomes}

Primary outcome

This was the proportion of total drinks selected that were alcoholic.

Secondary outcomes

Alcohol unit indices.

- Mean alcohol units from all drinks selected. This was assessed by dividing the total number of alcohol units selected by the total number of drinks selected [e.g. if two bottles of wine were selected (each 10 units) and two bottles of soft drink were selected: $20 / 4=5$ mean units of alcohol].

- Number of alcohol units selected. This was the total number of alcohol units contained within the selected alcohol products.

Alcohol quasi-purchasing indices.

- Total spend ( $f$ ) on alcoholic drinks. This was the total spend on alcoholic drinks.

- Spend ( $f$ ) on alcoholic drinks as a proportion of total spend. This was the total spend on alcoholic drinks.

Alcohol selection.

- Proportion of participants selecting at least one alcoholic beverage. This was a binary outcome of 'did not select an alcoholic beverage' or 'did select at least one alcoholic beverage' ( 0 or 1 , respectively).

Post intervention measures (assessed after the shopping task).

- Negative emotional arousal. This was assessed using the mean of a four-item measure, previously used to assess the impact of warning labels on cigarette packages [31] and adapted for alcohol HWL studies [20,23]. Responses were rated on 7-point scales: 'How (afraid/worried/uncomfortable/disgusted) does the label on this drink make you feel?' [1, not at all (afraid/worried/uncomfortable/ disgusted) to 7 , very (afraid/worried/uncomfortable/ disgusted)].

- Acceptability of health warning labels. This was assessed using one item on a 7-point scale, adapted from previous research assessing the impact of sugar tax [32] and alcohol HWLs [20]: 'Do you support or oppose putting this label on alcoholic drinks?' (Strongly oppose-neither oppose nor support-strongly support). Ratings past the scale mid-point (indicating neither acceptable nor unacceptable), i.e. $>4$, indicated that the label was acceptable.

\section{Procedure}

Ethical approval was granted by the Cambridge Psychology Research Ethics Committee (PRE.2019.058).

A pilot study was conducted $(n=10)$ to ensure that the setting and task were representative of a real shopping experience and that the task wording was clear.

Participants were selected from the research agency's existing pool of participants and were first invited by e-mail to complete an initial screening questionnaire. To disguise the true aim of the study, it was described as an experiment on 'shopping habits and behaviour' and the screening questionnaire contained filler questions on general shopping behaviours and snack consumption. If deemed fully eligible, participants were randomised to a specific HWL group and an appropriate time for study participation was arranged (see Randomisation section for details). Upon arrival, after providing informed consent, eligible participants answered questions regarding their demographic characteristics [age, gender, ethnicity, education (highest level), income], before completing the shopping task.

Participants were given the instructions: 'Imagine you are going to do your weekly shop with your own money, including buying drinks and snacks. Shop how you would usually shop for drinks and snacks, and if there is something that you would not be happy to buy, you do not have to buy it'. Participants were informed that all prices reflected typical market value and to imagine that they were spending their own money when making their selection (no minimum or maximum spend), but that they were not expected to purchase the items. Participants were given a shopping basket to browse the Shopper Lab aisles and then selected the products they wanted to purchase (this took no longer than 10 minutes). Depending on their allocated condition, the alcoholic drinks displayed an image-and-text HWL, text-only HWL or no HWLs (Box 1). In the HWL groups each alcoholic drink displayed one of six (image-and-text or text-only) different warnings on their packaging.

After the shopping task, participants left behind their selected items and were asked if they noticed any labels on the products. Participants who noticed labels were asked to describe them. In a separate room, all participants were then randomised to view one of the six text-only and one of the six image-and-text HWLs (in a counterbalanced order) on a beer or wine bottle (depending on their preference) and rated each HWL on negative emotional arousal 
and acceptability. Further demographic details-height, weight and drinking characteristics [Alcohol Use Disorders Identification Test (AUDIT-C) [33], weekly consumption]were recorded. Participants were debriefed, answered questions on the study aims and were given the opportunity to provide feedback. Participants were reimbursed $£ 20$ for their time.

\section{Statistical analysis (Supporting information, material S6)}

The primary outcome was the proportion of drinks selected that were alcoholic [number of alcoholic drinks divided by the total number of drinks (alcoholic plus non-alcoholic)]. This was analysed using a binomial generalised linear mixed model. The Bayes factor was calculated from the Bayesian information criteria for models with and without the experimental condition [34].

For the secondary outcomes of mean units of alcohol selected, total units of alcohol selected, total spend on alcoholic drinks and spend on alcoholic drinks as a proportion of total spend a generalised linear mixed model was used.
For the secondary outcome of the odds of selecting at least one alcoholic beverage, a logistic mixed model was used.

Negative emotional arousal and acceptability were analysed as difference scores using two separate general linear models. Adjustment was made for previous study group allocation by adding this variable as a covariate.

General free text comments about the HWLs or the study were coded and thematically analysed following the six steps outlined by Braun \& Clarke (2006) [35]. Themes were agreed between all authors.

\section{RESULTS}

In total, 399 participants were randomised, all of whom completed the study. Figure 1 shows the flow of participants through the study and Table 1 their characteristics across groups. Just over half the sample were female (55\%) and the mean age was 39.9 years [standard deviation $(\mathrm{SD})=13.7 ;$ median $=46 ;$ range $=24-61]$. Groups were well balanced on most characteristics. Due to concerns regarding differences between study groups of weekly consumption of alcohol (Table 1), and because it was

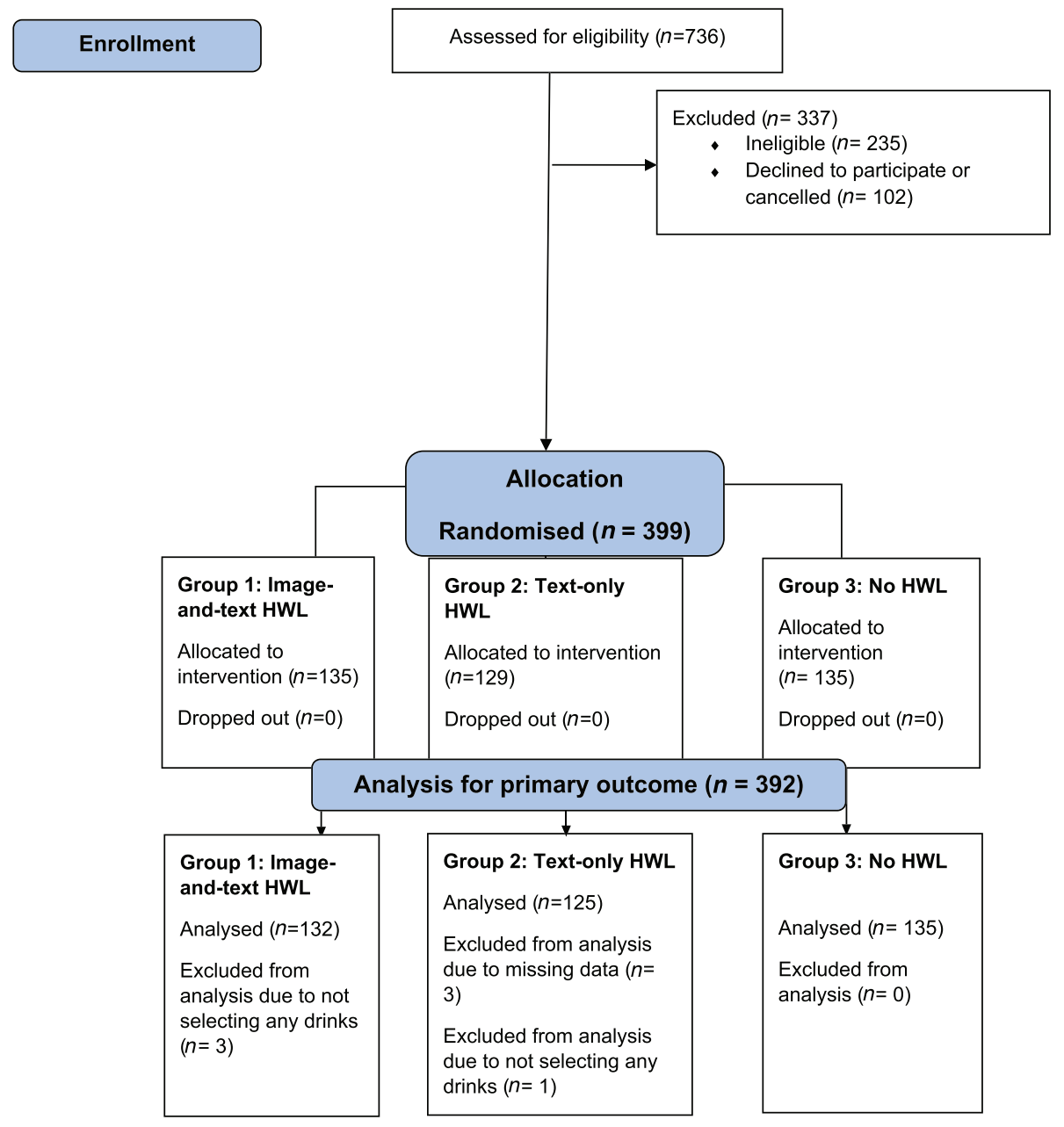

Figure I Flow of participants through study [Colour figure can be viewed at wileyonlinelibrary.com] 
Table 1 Participant characteristics ( $n \%$, unless otherwise stated).

\begin{tabular}{lll} 
Study condition & \\
\hline Group 1: Image-and-text HWL & Group 2: Text-only HWL & Group 3: Control (no HWL) \\
$n=135$ & $n=129$ & $n=135$
\end{tabular}

\begin{tabular}{|c|c|c|c|}
\hline \multicolumn{4}{|l|}{ Weekly alcohol consumption (units) ${ }^{\mathrm{a}}$} \\
\hline Mean (SD) & $15.6(11.9)$ & $17.5(11.9)$ & $19.4(15.9)$ \\
\hline $0-14$ & $82(61 \%)$ & $68(53 \%)$ & $62(46 \%)$ \\
\hline $15-30$ & $36(27 \%)$ & $45(35 \%)$ & $50(37 \%)$ \\
\hline $31-50$ & $16(12 \%)$ & $13(10 \%)$ & $16(12 \%)$ \\
\hline $51+$ & $1(<1 \%)$ & $3(2 \%)$ & $7(5 \%)$ \\
\hline AUDIT-C ${ }^{b}$ score (mean \pm SD) & $6.2(2.1)$ & $6.7(2.1)$ & $6.7(2.2)$ \\
\hline Age, mean (SD) & $40.4(13.6)$ & $39.0(13.9)$ & $40.3(13.5)$ \\
\hline $18-39$ years & $61(45 \%)$ & $67(52 \%)$ & $63(47 \%)$ \\
\hline $40-59$ years & $55(41 \%)$ & $41(32 \%)$ & $53(39 \%)$ \\
\hline 60 and over & $19(14 \%)$ & $21(16 \%)$ & $19(14 \%)$ \\
\hline \multicolumn{4}{|l|}{ Gender } \\
\hline Male & $59(44 \%)$ & $64(50 \%)$ & $57(42 \%)$ \\
\hline Female & $76(56 \%)$ & $65(50 \%)$ & $78(58 \%)$ \\
\hline \multicolumn{4}{|l|}{ Ethnicity } \\
\hline White & $131(97 \%)$ & $120(93 \%)$ & $127(94 \%)$ \\
\hline Mixed & $3(2 \%)$ & $2(2 \%)$ & $5(4 \%)$ \\
\hline Asian & $0(0 \%)$ & $4(3 \%)$ & $2(2 \%)$ \\
\hline Black & $1(<1 \%)$ & $3(2 \%)$ & $1(<1 \%)$ \\
\hline \multicolumn{4}{|l|}{ Highest qualification } \\
\hline No qualifications & $4(3 \%)$ & $7(5 \%)$ & $3(2 \%)$ \\
\hline Up to 4 GCSEs & $24(18 \%)$ & $34(26 \%)$ & $41(30 \%)$ \\
\hline 5 or more GCSEs or 1 A-level & $43(32 \%)$ & $31(24 \%)$ & $32(24 \%)$ \\
\hline 2 or more A-levels & $23(17 \%)$ & $19(15 \%)$ & $20(15 \%)$ \\
\hline Bachelor's degree & $25(18 \%)$ & $20(16 \%)$ & $26(19 \%)$ \\
\hline Post-graduate degree or qualification & $16(12 \%)$ & $18(14 \%)$ & $13(10 \%)$ \\
\hline
\end{tabular}

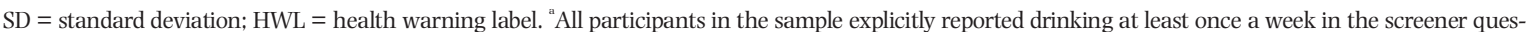
tions. A further weekly drinking measure recorded the amount of alcohol consumed in the previous week as an overall indication of the volume of alcohol consumed weekly. 'Heavy and binge drinking behaviours [Alcohol Use Disorders Identification Test (AUDIT-C) [33]], three questions to detect heavy and binge drinking behaviour in a general population, with a total score of 0 (low risk) to 12 (high risk) [33].

highly related to the outcome, this measure was added as a continuous covariate to the primary outcome analysis, although its inclusion did not alter conclusions. Three participants were excluded from primary and secondary outcome analysis due to missing data.

\section{Primary outcome (see Tables 2 and 3)}

Compared to no HWL, modelling suggested no clear evidence of a difference in the HWL groups for the percentage of drinks selected that were alcoholic: image-and-text HWL: 46\%; text-only HWL: 41\%; no HWL: 44\% (adjusted values, see Table 2 for unadjusted means). Concordant with there being no difference between groups in this specific study context, there was extreme evidence in favour of the null hypothesis [Bayes factor $(\mathrm{BF})<0.01]$.

\section{Secondary outcomes}

Raw secondary outcome data are presented in Table 2 and modelled estimates in Table 3. Compared to no HWL, modelling suggested no clear evidence of a difference in the HWL groups for any of the secondary outcomes.

HWL ratings (Table 4)

Negative emotional arousal ratings were higher in the image-and-text HWL group compared to the text-only HWL group. Acceptability was lower in the image-and-text HWL group compared to the text-only HWL group.

Based on a score of 5 or more on the 7-point scale, $53 \%$ $(n=213)$ of participants indicated that they supported text-only HWLs and 41\% $(n=163)$ supported imageand-text HWLs. 
Table 2 Primary and secondary outcomes [unadjusted mean $^{\mathrm{a}}(\mathrm{SD})$ and $n \%$ ].

\begin{tabular}{|c|c|c|c|}
\hline & $\begin{array}{l}\text { Group 1: Image-and-text HWL } \\
n=135\end{array}$ & $\begin{array}{l}\text { Group 2: Text-only HWL } \\
n=126\end{array}$ & $\begin{array}{l}\text { Group 3: Control (no HWL) } \\
n=135\end{array}$ \\
\hline & \multicolumn{3}{|l|}{ Mean (SD) } \\
\hline Primary outcome: proportion of alcoholic drinks ${ }^{\mathrm{a}}$ & $0.50(0.29)$ & $0.46(0.26)$ & $0.45(0.28)$ \\
\hline \multicolumn{4}{|l|}{ Secondary outcomes } \\
\hline Mean number of alcohol units selected ${ }^{\mathrm{b}}$ & $3.33(2.71)$ & $2.99(2.54)$ & $3.12(3.05)$ \\
\hline Total number of alcohol units selected & $12.23(9.93)$ & $11.93(8.92)$ & $12.33(12.04)$ \\
\hline Total spend on alcoholic drinks, in $£$ & $9.09(7.03)$ & $10.52(11.68)$ & $9.56(9.87)$ \\
\hline \multirow[t]{2}{*}{$\begin{array}{l}\text { Total spend on alcoholic drinks as a proportion } \\
\text { of total spend }{ }^{\text {a }}\end{array}$} & $0.68(0.30)$ & $0.68(0.28)$ & $0.66(0.32)$ \\
\hline & $n(\%)$ & & \\
\hline $\begin{array}{l}\text { Number of participants who selected at least one } \\
\text { alcoholic drink }\end{array}$ & $117(87)$ & $114(91)$ & $114(84)$ \\
\hline
\end{tabular}

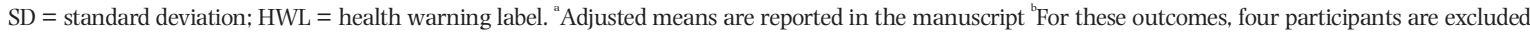
(three in the image-and-text HWL group, one in the text-only HWL group), as they did not select any drinks, i.e. these outcomes would have been calculated as $0 / 0)$.

Table 3 Estimated mean differences $(95 \%$ CI) or odds ratios $(95 \%$ CI) for HWL groups compared to no HWL $(n=135)$ for primary and secondary outcomes.

\begin{tabular}{|c|c|c|}
\hline & Group 1: Image-and-text HWL $n=135$ & Group 2: Text-only HWL $n=126$ \\
\hline & \multicolumn{2}{|l|}{ OR (95\% CI), P-value } \\
\hline Primary outcome: proportion of alcoholic drinks ${ }^{a, b}$ & $1.08(0.82,1.42), P=0.55$ & $0.87(0.67,1.14), P=0.31$ \\
\hline Secondary outcomes & \multicolumn{2}{|l|}{ MD (95\% CI), P-value, effect size (Cohen's d) ${ }^{c}$} \\
\hline Mean number of alcohol units selected ${ }^{\mathrm{a}}$ & $\begin{array}{l}0.22(-0.47,0.91) \\
P=0.53, d=0.07\end{array}$ & $\begin{array}{l}-0.13(-0.82,0.56) \\
P=0.71, d=-0.04\end{array}$ \\
\hline Total number of alcohol units selected & $\begin{array}{l}-0.09(-2.65,2.48) \\
P=0.95, d=-0.01\end{array}$ & $\begin{array}{l}-0.37(-2.90,2.16) \\
P=0.77, d=-0.04\end{array}$ \\
\hline Total spend on alcoholic drinks, in $£$ & $\begin{array}{l}-0.43(-2.41,1.56) \\
P=0.67, d=-0.05\end{array}$ & $\begin{array}{l}1.15(-1.43,3.72) \\
P=0.38, d=0.11\end{array}$ \\
\hline \multirow[t]{2}{*}{$\begin{array}{l}\text { Total spend on alcoholic drinks as a } \\
\text { proportion of total spend }^{\text {a }}\end{array}$} & $\begin{array}{l}0.03(-0.04,0.11) \\
P=0.36, d=0.09\end{array}$ & $\begin{array}{l}0.01(-0.06,0.09) \\
P=0.73, d=0.03\end{array}$ \\
\hline & OR (95\% CI), P-value & \\
\hline $\begin{array}{l}\text { Number of participants who selected at } \\
\text { least one alcoholic drink }\end{array}$ & $0.85(0.42,1.70), P=0.64$ & $0.63(0.28,1.42), P=0.27$ \\
\hline
\end{tabular}


Table 4 Estimated mean differences $(95 \%$ CI) for negative emotional arousal and acceptability ratings—within subjects $(n=399)$.

\begin{tabular}{lllll}
\hline & Raw mean (SD) & Estimated MD $(95 \% \text { CI })^{a}$ & P-value $^{a}$ & t-statistic (d.f. $)^{a}$ \\
\hline Negative emotional arousal & & & & \\
$\quad$ Image-and-text HWL & $4.29(1.67)$ & $1.04(0.87,1.22)$ & - & $t(396)=11.72$ \\
$\quad$ Text-only HWL & $3.25(1.46)$ & - & & \\
Acceptability & & & -0.001 & $t(396)=-5.15$ \\
$\quad$ Image-and-text HWL & $3.64(1.95)$ & - & -0.001 & \\
$\quad$ Text-only HWL & $4.32(1.74)$ & & &
\end{tabular}

$\mathrm{CI}=$ confidence interval; d.f. $=$ degrees of freedom; $\mathrm{HWL}=$ health warning labels; $\mathrm{MD}=$ mean difference; $\mathrm{SD}=$ standard deviation. ${ }^{\mathrm{a}}$ Estimated using a general linear model of the difference scores between image-and-text and text-only, with adjustment for previous study group allocation and clustering (18-level session variable) as a nested random variable.

Table 5 Themes identified in free-text comments.

\begin{tabular}{lll}
\hline Theme & Subtheme & Description
\end{tabular}

Theme 1: Drinkers' position on the acceptability of HWLS

General
support for
HWLs

General opposition to HWLs

Unintended consequences

Drinking context and culture
Theme 2: Potential How people impact of the HWLs behave
Health warning labels (HWLs) were received positively and described as a good idea; for example, participants mentioned that they were necessary or even thought they should be a legal requirement. Some said they should be used because they have worked on tobacco. Some thought only the text-only HWLs were acceptable

HWLs were received negatively and described as a bad idea. Generally, images were less accepted than text-only HWLs. References to nanny-state and that drinking should be an individual choice. Terms such as 'unnecessary', 'patronising' and 'scaremongering' used HWLs may have unintended consequences, such as a negative impact on children exposed to them, increased stigma or judgement potentially leading to depression, shame or anxiety around drinking. The potential negative impact on sales in pubs and small alcohol businesses were also mentioned as an adverse effect of the labels References to the value of alcohol in society, as a part of culture. HWLs as suitable for certain types of drinks (such as cheap, strong, bulk buys), but not for premium products (such as wine, real ales or an expensive whisky). Drinking was highlighted as a social and enjoyable activity, which participants did not want ruined by the labels. Many participants expressed a sense of unfairness that their responsible and 'civilised' drinking would be punished, when in their eyes it is specific contexts of drinking, e.g. binge drinking, which should be targeted. Similarly, participants described that alcohol is often given as gifts or consumed as social events in pubs or bars

Participants highlighted they perceived HWLs 'I think labels would make me drink less' as being effective in deterring themselves or
'I personally do not like them and feel we are in danger of living in a nanny state'

'I feel products should have warning labels on but the images are too far, as a parent I would not feel comfortable with my children seeing images like this in the supermarket'

'I find them distasteful. A bottle of wine is a beautiful thing and is spoilt by them'$$
\text { 'mages like this in the supermarke' }
$$

'They are a great idea'

'I think labels would make me drink less' 
Table 5. (Continued)

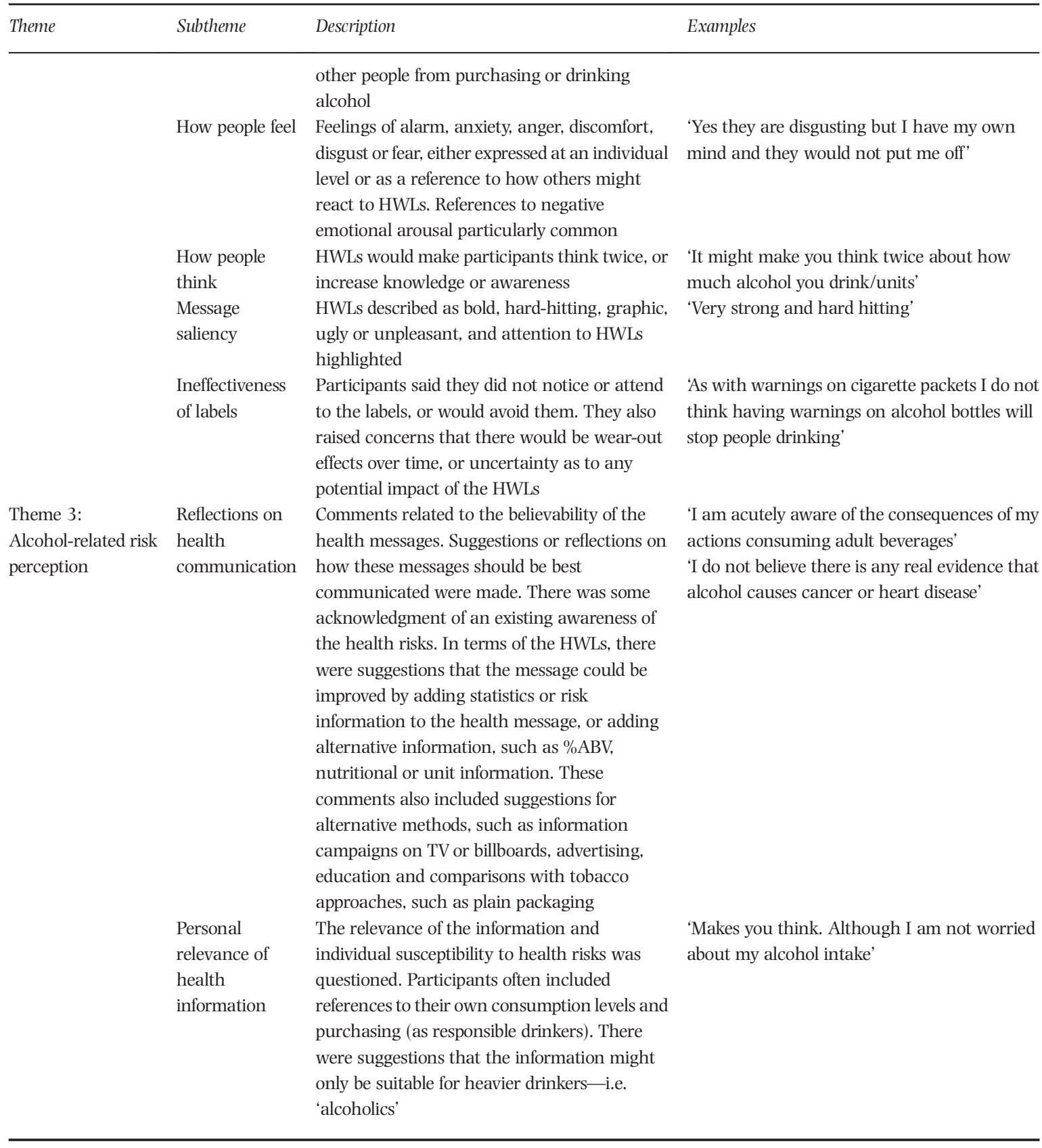

\section{Additional analyses}

See Supporting information, S7 for additional analyses including sensitivity analyses and thematic analysis (themes in Table 5). Supporting information, Table S7d shows a model that includes participant characteristics as covariates, with conclusions unchanged.

\section{DISCUSSION}

In a naturalistic shopping laboratory, health warning labels (HWLs) describing the adverse health consequences of excessive alcohol consumption did not change selection behaviour. There was no clear evidence of a difference between groups for the primary outcome - the proportion of alcoholic drinks selected-or for any of the secondary outcome measures.

These findings are inconsistent with prior studies that show promising effects of alcohol HWLs on selection in online settings using identical HWLs [20], on sales in field settings [13] and on consumption in laboratory settings [21]. They are also not consistent with evidence that suggests HWLs on other products, such as sugary drinks, can change purchasing behaviour in a similar shopping laboratory context [29]. 
There are four possible explanations for the current findings. First, HWLs could impact selection in a real-world setting, but the shopping laboratory setting and study task lacked ecological validity. While the quasi-purchasing task used in the current study was equivalent to the standard task used widely in the commercial sector and the majority of participants indicated that the shopping task felt at least somewhat typical compared to their usual shop, we are unaware of any evidence in the public domain that directly tests its validity. In contrast to other studies conducted in similar settings [29], participants did not exchange money or keep selected items. This was not possible in the present study, as alcohol cannot be sold in unlicensed premises [36]. The exchange of money could equally have introduced other biases, such as choices based on product prices. For example, another study using a similar design [29] provided an incentive to each participant to purchase products and the participant kept the remaining money. Depending on the price of the products and the value participants attached to cash, this might have increased or decreased purchasing of the study products.

A second possibility related to the study task is that HWLs may impact more opportunistic behaviours, such as selecting an item for immediate consumption, as typically measured in online settings [20,37], rather than as part of a typical weekly shop. This may have encouraged relatively routine or planned shopping behaviour, where participants were motivated to select products that they usually purchased (other studies showing effects on sugary drink purchasing have not specified a weekly shop in task wording [29,38]). Such behaviour may be less likely to be influenced by environmental cues such as packaging.

A third possible explanation for the null effect in the current study is that a brief exposure to HWLs placed on real alcohol products at a single time-point does not affect selection or has an effect that the current study was underpowered to detect. While the majority of participants indicated that they noticed the HWLs, future studies could assess differences in responses to HWLs in greater detail by measuring viewing time. The evidence that HWLs on sugary drinks can have an immediate impact on selection in a similar setting [29] raises the possibility that responses to HWLs on alcohol may differ from responses to HWLs on other products [39]. We should be cautious when comparing across products, as they are different substances associated with specific behaviours, even though alcohol shares some properties with both sugary drinks (foodstuff) and tobacco (drug) [11]. Moreover, our findings do not obviate the possibility that repeated exposure to HWLs could impact behaviour over the longer term, as has been observed in multiple studies in tobacco $[6,40,41]$. It is also possible that HWLs could affect other drinking behaviours, such as the rate at which alcohol is consumed [21].
Finally, the ineffectiveness of the HWLs in changing selection in the current study could be due to participants being relatively light drinkers: just over half the participants in the current study reported drinking within the low-risk drinking guideline amount of up to 14 units per week [42]. However, this is a similar sample in terms of drinking characteristics to our previous online study, where HWLs reduced selection [20] and AUDIT-C scores of the majority of the sample $(83 \%)$ are indicative of more risky drinking (score of 5+). An exploratory analysis also found that including participants' characteristics as covariates-including alcohol consumption-did not change model conclusions. While drinkers at all levels of risk should be aware of potential harms [43], there may be differences in responses to HWLs by consumption or perceived relevance of health harms, warranting further study.

Despite there being no impact upon selection behaviour, HWLs affected emotions and cognitions. Negative emotional arousal was higher, and acceptability lower, in the image-and-text HWL group, compared to the text-only HWL group, mirroring findings from online settings [20]. Negative emotional arousal has been shown to be a potential mediator of the effect of HWLs on selection of alcohol and other products in online settings [20,37,44]. In the current setting it may be that increases in negative emotional arousal were insufficient, or other conditions are required, for an effect upon selection behaviour to be realised.

Approximately half the sample (53\%) perceived text-only HWLs as acceptable, and this was lower (41\%) for image-and-text HWLs. In free-text comments, some participants advocated their implementation and others thought they were unacceptable, in accordance with other studies that suggest mixed acceptability for alcohol HWLs $[23,45]$. Acceptability of alcohol policies may be higher with an increase in knowledge of health harms, specifically that alcohol can cause cancer [24]. Acceptability of an intervention, such as HWLs, is also higher when evidence for its effectiveness is communicated [46].

Thematic analysis of free-text comments highlighted that participants perceived HWLs as having potential effects on other cognitions including awareness, attention and knowledge. The evidence as to whether these changes lead to consistent changes in behaviour is variable. Previous research suggests that alcohol HWLs may improve recall and health-related knowledge [47], and such cognitions may be precursors to behaviour change [40,48]. However, labelling on other products has been found to change cognitions (e.g. implicit attitudes), but these changes are not necessarily associated with changes in behaviour [49,50]. Future research should investigate whether, when and under which conditions such cognitions and emotions translate to behaviour change. Furthermore, knowledge of the alcohol-cancer link is low [19,51], and many participants in the current study expressed surprise at the depicted 
health risks. As such, changes in cognitions are potentially positive outcomes of HWLs in their own right.

Free-text comments also highlighted that the HWLs used in the present study may be suitable for heavy or problem drinkers, but not for 'sensible' drinkers such as themselves - an 'othering' effect which has been observed in other qualitative studies of alcohol interventions [52]. This emphasises the need to communicate health consequences associated with drinking at all consumption levels, together with low-risk drinking guidelines. References to alcohol's particular cultural significance to participants, and the pleasure obtained from drinking, were also common. This aligns with previous research highlighting the importance of considering the culture of drinking and different drinking practices when assessing the effectiveness of potential interventions [53]. Additional in-depth qualitative work could further illuminate the apparent ineffectiveness of the HWLs.

\section{Strengths and limitations}

This pre-registered, randomised study is the first to investigate alcohol HWLs in a commercial-standard naturalistic shopping laboratory setting, with HWLs displayed across a wide selection of real products. It also has some limitations.

First, although real products were used, participants did not keep the products or exchange money. Although commonplace in such settings for commercial research, as discussed above, this may have decreased ecological validity. In addition, participants were aware that they were being observed, which may have further affected ecological validity and potentially altered behaviour. Secondly, given that our sample size was based on effect sizes from an online setting [20], it is possible the study was underpowered. However, the results do not suggest effects in the expected direction and the Bayes factor suggests extreme evidence for no difference between groups.

\section{Implications for future research}

Our findings provide limited evidence that HWLs on alcohol may not be effective in altering selection in a naturalistic setting. While actual purchasing may elicit its own biases, on balance we judge that stronger evidence would be provided by studies that use objective purchasing measures-i.e. involving exchange of participants' own money - with products selected for consumption.

This study provides further evidence that effects of HWLs on real products may differ to effects seen in online studies using images of products [25]. Testing these interventions in naturalistic or field settings comes with clear challenges, as recently evidenced in Canada, where researchers were forced to withdraw cancer labels from alcohol products due to industry pressure [54]. HWLs are likely to be unpalatable to commercial stakeholders and wider industry pressures can be expected if such labels are considered for implementation.

Should further research confirm that HWLs are likely ineffective in the immediate term, they still have potential to be effective in the longer term, both directly through repeated exposure to product packaging, or indirectly, such as by resulting in changes to industry behaviours, e.g. through increased availability of lower strength or alcohol-free products that would not require HWLs. Such industry responses have been seen, for example, in relation to the UK sugar levy resulting in widespread re-formulation of sugary drinks to avoid crossing the taxable threshold for sugar content [55] and following the implementation of food labelling interventions [56].

\section{Conclusions}

In a naturalistic shopping laboratory, there was no evidence that health warning labels reduced selection of alcoholic drinks. These types of labels applied to alcohol products may be ineffective in changing selection behaviour, or the study as designed was unable to detect their effect.

\section{Clinical trial registration}

ISRCTN18275963; OSF: https://osf.io/hbdg3/.

\section{Declaration of interests}

None.

\section{Acknowledgements}

This research was funded in whole, or in part, by the Wellcome Trust (ref: 206853/Z/17/Z). For the purpose of Open Access, the author has applied a CC BY public copyright licence to any Author Accepted Manuscript version arising from this submission.

\section{Data availability statement}

The dataset generated and analysed during the current study are available on the Open Science Framework project page: https://osf.io/j9b63/ and the University of Cambridge Research Repository: https://doi. org/10.17863/CAM.66856.

\section{References}

1. Rehm J., Guiraud J., Poulnais R., Shield K. D. Alcohol dependence and very high risk level of alcohol consumption: a life-threatening and debilitating disease. Addict Biol 2018; 23: 961-8.

2. Sheron N., Gilmore I. Effect of policy, economics, and the changing alcohol marketplace on alcohol related deaths in England and Wales. BMJ Clin Res 2016; 353: i1860. 
3. World Health Organization (WHO). Global strategy to reduce the harmful use of alcohol. 2015. Available at: https://www. who.int/publications/i/item/9789241599931

4. Hammond D. Tobacco Labelling and Packaging Toolkit: A guide to FCTC Article 11. 2009; Available at: https://www. who.int/fctc/guidelines/adopted/article_11/en/. Accessed September 2020.

5. Hammond D., McDonald P., Fong G., Stephen Brown K., Cameron R. The impact of cigarette warning labels and smoke-free bylaws on smoking cessation: evidence from former smokers. Can J Public Health 2003; 95: 201-4.

6. Brewer N. T., Hall M. G., Noar S. M., Parada H., Stein-Seroussi A., Bach L. E., et al. Effect of pictorial cigarette packwarnings on changes in smoking behavior a randomised clinical trial. JAMA Intern Med 2016; 176: 905-12.

7. Hammond D. Health warning messages on tobacco products: a review. Tob Control 2011; 20: 327-37.

8. Noar S. M., Hall M. G., Francis D. B., Ribisl K. M., Pepper J. K., Brewer N. T. Pictorial cigarette pack warnings: a meta-analysis of experimental studies. Tob Control 2016; 25: 341-54.

9. Thrasher J. F., Carpenter M. J., Andrews J. O., Gray K. M., Alberg A. J., Navarro A., et al. Cigarette warning label policy alternatives and smoking-related health disparities. Am J Prev Med 2012; 43: 590-600.

10. Blackwell A. K. M., Drax K., Attwood A. S., Munafò M. R., Maynard O. M. Informing drinkers: can current UK alcohol labels be improved? Drug Alcohol Depend 2018; 192: 163-70.

11. Martin-Moreno J. M., Harris M. E., Breda J., Møller L., AlfonsoSanchez J. L., Gorgojo L. Enhanced labelling on alcoholic drinks: reviewing the evidence to guide alcohol policy. Eur J Public Health 2013; 23: 1082-7.

12. Royal Society for Public Health (RSPH). Labelling the point: towards better alcohol health information. 2018. Available at: https://www.rsph.org.uk/our-work/policy/drugs/labelling-the-point.html. Accessed September 2020.

13. Zhao J., Stockwell T., Vallance K., Hobin E. The effects of alcohol warning labels on population alcohol consumption: an interrupted time series analysis of alcohol sales in Yukon, Canada. J Stud Alcohol Drugs 2020; 81: 225-37.

14. Food Standards Agency. Food Standards Agency-Food labelling e-learning course. Available at: https:// labellingtraining.food.gov.uk/module10/overview_1.html. Accessed September 2020.

15. Portman Group. Alcohol Marketing and Sponsorship. 2019. Available at: https://www.portmangroup.org.uk/marketingtoolkit/. Accessed September 2020.

16. Kersbergen I., Field M. Alcohol consumers' attention to warning labels and brand information on alcohol packaging: findings from cross-sectional and experimental studies. BMC Public Health 2017; 17: 123.

17. Burton R., Henn C., Lavoie D., O'Connor R., Perkins C., Sweeney K., et al. A rapid evidence review of the effectiveness and cost-effectiveness of alcohol control policies: an English perspective. Lancet 2017; 389: 1558-80.

18. Jané-Llopis E, Kokole D, Neufeld M, Hasan OSM, Rehm J. WHO Health Evidence Network Synthesis Report 68. 2020;112. Available at: https://apps.who.int/iris/bitstream/ handle/10665/332129/9789289054898-eng.pdf?ua=1

19. Buykx P. An investigation of public knowledge of the link between alcohol and cancer. BMC Public Health 2015; 16: 1194.
20. Clarke N., Pechey E., Mantzari E., Blackwell A. K. M., De-loyde K., Morris R. W., et al. Impact of health warning labels communicating the risk of cancer on alcohol selection: an online experimental study. Addiction 2021; 116: $41-52$.

21. Stafford L. D., Salmon J. Alcohol health warnings can influence the speed of consumption. J Public Health 2017; 25: 147-54.

22. Wigg S., Stafford L. D. Health warnings on alcoholic beverages: perceptions of the health risks and intentions towards alcohol consumption. PLOS ONE 2016; 4: e0153027.

23. Pechey E., Clarke N., Mantzari E., Blackwell A. K. M., De-Loyde K., Morris R. W., et al. Image-and-text health warning labels on alcohol and food: potential effectiveness and acceptability. BMC Public Health 2020; 20: 376.

24. Weerasinghe A., Schoueri-Mychasiw N., Vallance K., Stockwell T., Hammond D., McGavock J., et al. Improving knowledge that alcohol can cause cancer is associated with consumer support for alcohol policies: findings from a realworld alcohol labelling study. Int J Environ Res Public Health 2020; 17: -398.

25. Clarke N., Pechey E., Kosite D., König L. M., Mantzari E., Blackwell A. K. M., et al. Impact of health warning labels on selection and consumption of food and alcohol products: systematic review with meta-analysis. Health Psychol Rev 2020; 1-39.

26. Kersbergen I., Oldham M., Jones A., Field M., Angus C., Robinson E. Reducing the standard serving size of alcoholic beverages prompts reductions in alcohol consumption. Addiction 2018; 113: 1598-608.

27. Setodji C. M., Martino S. C., Gong M., Dunbar M. S., Kusuke D., Sicker A., et al. How do tobacco power walls influence adolescents? A study of mediating mechanisms. Health Psychol 2018; 37: 188-93.

28. Shadel W. G., Martino S. C., Setodji C. M., Dunbar M., Scharf D., Creswell K. G. Do graphic health warning labels on cigarette packages deter purchases at point-of-sale? An experiment with adult smokers. Health Educ Res 2019; 34: 321-31.

29. Grummon A., Taillie L. S., Golden S., Hall M., Ranney L., Brewer N. Impact of sugar-sweetened beverage health warnings on beverage purchases: a randomised controlled trial (OR28-01-19). Curr Dev Nutr 2019; nzz042. OR28-01-19.

30. Hollands G. J., Bignardi G., Johnston M., Kelly M. P., Ogilvie D., Petticrew M., et al. The TIPPME intervention typology for changing environments to change behaviour. Nat Hum Behav 2017; 1: 0140 .

31. Kees J., Burton S., Craig Andrews J., Kozup J. Understanding how graphic pictorial warnings work on cigarette packaging. J Public Policy Mark 2010; 29: 265-76.

32. Reynolds J. P., Archer S., Pilling M., Kenny M., Hollands G. J., Marteau T. M. Public acceptability of nudging and taxing to reduce consumption of alcohol, tobacco, and food: a population-based survey experiment. Soc Sci Med 2019; 236: 112395.

33. Bush K., Kivlahan D. R., McDonell M. B., Fihn S. D., Bradley K. A. The AUDIT alcohol consumption questions (AUDIT-C): an effective brief screening test for problem drinking. Ambulatory care quality improvement project (ACQUIP). Alcohol use disorders identification test. Arch Intern Med 1998; 158: 1789-95. 
34. Lindeløv JK. How to compute Bayes factors using $\mathrm{lm}$, Imer, BayesFactor, brms, and JAGS/stan/pymc3. 2018. Available at: https://vbn.aau.dk/da/publications/how-to-computebayes-factors-using-lm-lmer-bayesfactor-brms-and-j

35. Braun V., Clarke V. Using thematic analysis in psychology. Qual Res Psychol 2006; 3: 77-101.

36. Institute of Alcohol Studies. Licensing Laws. Available at: http://www.ias.org.uk/Alcohol-knowledge-centre/Availability-and-licensing/Factsheets/Licensing-laws.aspx. Accessed September 2020.

37. Mantzari E., Vasiljevic M., Turney I., Pilling M., Marteau T. Impact of warning labels on sugar-sweetened beverages on parental selection: an online experimental study. Prev Med Rep 2018; 12: 259-67.

38. Donnelly G. E., Zatz L. Y., Svirsky D., John L. K. The effect of graphic warnings on sugary-drink purchasing. Psychol Sci 2018; 29: 1321-33.

39. Hall M. G., Grummon A. H., Lazard A. J., Maynard O. M., Taillie L. S. Reactions to graphic and text health warnings for cigarettes, sugar-sweetened beverages, and alcohol: an online randomised experiment of US adults. Prev Med 2020; 106120.

40. Hammond D., Fong G. T., McDonald P. W., Cameron R., Brown K. S. Impact of the graphic Canadian warning labels on adult smoking behaviour. Tob Control 2003; 12: 391-5.

41. Schüz N., Eid M., Schüz B., Ferguson S. G. Immediate effects of plain packaging health warnings on quitting intention and potential mediators: results from two ecological momentary assessment studies. Psychol Addict Behav 2016; 30: 220-8.

42. Department of Health. UK Chief Medical Officers' Alcohol Guidelines Review. Summary of the proposed new guidelines. 2016. Available at: https://assets.publishing.service.gov.uk/ government/uploads/system/uploads/attachment_data/file/ 489795/summary.pdf. Accessed September 2020.

43. Sherk A., Thomas G., Churchill S., Stockwell T. Does drinking within low-risk guidelines prevent harm? Implications for high-income countries using the international model of alcohol harms and policies. J Stud Alcohol Drugs 2020; 81: 352-61.

44. Cho Y. J., Thrasher J. F., Yong H.-H., Szklo A. S., O'Connor R. J., Bansal-Travers M., et al. Path analysis of warning label effects on negative emotions and quit attempts: a longitudinal study of smokers in Australia, Canada, Mexico, and the US. Soc Sci Med 2018; 197: 226-34.

45. Vallance K., Stockwell T., Zhao J., Shokar S., SchoueriMychasiw N., Hammond D., et al. Baseline assessment of alcohol-related knowledge of and support for alcohol warning labels among alcohol consumers in northern Canada and associations with key sociodemographic characteristics. J Stud Alcohol Drugs 2020; 81: 238-48.

46. Reynolds J. P., Stautz K., Pilling M., van der Linden S., Marteau T. M. Communicating the effectiveness and ineffectiveness of government policies and their impact on public support: a systematic review with meta-analysis. $R$ Soc Open Sci 2020; 7: 190522.

47. Hobin E., Weerasinghe A., Vallance K., Hammond D., McGavock J., Greenfield T. K., et al. Testing alcohol labels as a tool to communicate cancer risk to drinkers: a real-world quasi-experimental study. J Stud Alcohol Drugs 2020; 81: 249-61.

48. Brewer N. T., Parada H., Hall M. G., Boynton M. H., Noar S. M., Ribisl K. M. Understanding why pictorial cigarette pack warnings increase quit attempts. Ann Behav Med 2019; 53 232-43.
49. Asbridge S. C. M., Pechey E., Marteau T. M., Hollands G. J. Effects of pairing health warning labels with energy-dense snack foods on food choice and attitudes: online experimental study. Appetite 2021; 160: 105090.

50. Crockett R. A., King S. E., Marteau T. M., Prevost A. T., Bignardi G., Roberts N. W., et al. Nutritional labelling for healthier food or non-alcoholic drink purchasing and consumption. Cochrane Database Syst Rev 2018; 2: CD009315.

51. Winstock A. R., Holmes J., Ferris J. A., Davies E. L. Perceptions of alcohol health warning labels in a large international cross-sectional survey of people who drink alcohol. Alcohol Alcohol 2020; 16;55: 315-22.

52. Bareham B. K., Kaner E., Spencer L. P., Hanratty B. Drinking in later life: a systematic review and thematic synthesis of qualitative studies exploring older people's perceptions and experiences. Age Ageing 2019; 48: 134-46.

53. Ally A. K., Lovatt M., Meier P. S., Brennan A., Holmes J. Developing a social practice-based typology of British drinking culture in 2009-2011: implications for alcohol policy analysis. Addiction 2016; 111: 1568-79.

54. Stockwell T., Solomon R., O'Brien P., Vallance K., Hobin E. Cancer warning labels on alcohol containers: a Consumer's right to know, a Government's responsibility to inform, and an Industry's power to thwart. J Stud Alcohol Drugs 2020; 81: 284-92.

55. Scarborough P., Adhikari V., Harrington R. A., Elhussein A., Briggs A., Rayner M., et al. Impact of the announcement and implementation of the UK soft drinks industry levy on sugar content, price, product size and number of available soft drinks in the UK, 2015-19: a controlled interrupted time series analysis. PLOS Med 2020; 17: e1003025.

56. Shangguan S., Afshin A., Shulkin M., Ma W., Marsden D., Smith J., et al. A meta-analysis of food labeling effects on consumer diet behaviors and industry practices. Am J Prev Med 2019; 56: 300-14.

\section{Supporting Information}

Additional supporting information may be found online in the Supporting Information section at the end of the article.

Figure S1. Study flow diagram with randomisations.

Figure S2. Session allocation - randomisation procedure.

Figure S3. Shopper Lab layout.

Figure S4. Images of the shopping laboratory.

Figure S5. Example HWLs.

Table S7a. Sensitivity analysis 1. Manipulation check for primary outcome - proportion of drinks selected that were alcoholic $(n=307)$.

Table S7b. Sensitivity analysis 2. Participants identified by onsite research agency team for primary outcome - proportion of drinks selected that were alcoholic $(n=386)$.

Table S7c. Estimated means (robust SEs) for continuous secondary outcomes $(n=396)$.

Table S7d. Exploratory analysis. Updated model results with additional covariates included - primary outcome. 